\title{
Electronic friction and liquid-flow-induced voltage in nanotubes
}

\author{
B. N. J. Persson, ${ }^{1,2,3}$ U. Tartaglino, ${ }^{4}$ E. Tosatti, ${ }^{3,4,5}$ and H. Ueba ${ }^{6}$ \\ ${ }^{1}$ IFF, FZ-Jülich, 52425 Jülich, Germany \\ ${ }^{2}$ Kavli Institute for Theoretical Physics, University of California, Santa Barbara, California 93106-4030, USA \\ ${ }^{3}$ International Centre for Theoretical Physics (ICTP), P.O. Box 586, I-34014 Trieste, Italy \\ ${ }^{4}$ International School for Advanced Studies (SISSA), and INFM Democritos National Simulation Center, Via Beirut 2 , \\ I-34014 Trieste, Italy \\ ${ }^{5}$ Laboratoire de Mineralogie-Cristallographie de Paris, Université Pierre et Marie Curie, 4 place Jussieu, 75252 Paris Cedex, France \\ ${ }^{6}$ Department of Electronics, Toyama University, Gofuku, Toyama, 930-8555, Japan
}

(Received 14 January 2004; published 25 June 2004)

\begin{abstract}
A recent exciting experiment by Ghosh et al. [Science 299, 1042 (2003)] reported that the flow of an ion-containing liquid such as water through bundles of single-walled carbon nanotubes induces a voltage in the nanotubes that grows logarithmically with the flow velocity $v_{0}$. We propose an explanation for this observation. Assuming that the liquid molecules nearest the nanotube form a two-dimensional solidlike monolayer pinned through the adsorbed ions to the nanotubes, the monolayer sliding will occur by elastic loading followed by the local yield (stick-slip motion). The drifting adsorbed ions produce a voltage in the nanotube through electronic friction against free electrons inside the nanotube. Thermally excited jumps over force-biased barriers, well known in the stick-slip model, can explain the logarithmic voltage growth with flow velocity. We estimate the short-circuit current and the internal resistance of the nanotube voltage generator.
\end{abstract}

DOI: 10.1103/PhysRevB.69.235410

PACS number(s): 72.80.Rj, 73.63.Fg

\section{INTRODUCTION}

In a recent, remarkable experiment, ${ }^{1}$ it was observed that the flow of an ion-rich liquid such as water through bundles of single-walled carbon nanotubes induces a voltage in the nanotube sample along the direction of the flow. Strikingly, the voltage grows logarithmically with the liquid flow velocity over nearly six decades. The voltage magnitude and sign depend sensitively on the ionic conductivity and on the polar nature of the liquid, suggesting that ions adsorbed or located in the immediate vicinity of the carbon nanotubes must be involved in its generation. This work highlights the device potential for nanotubes as sensitive flow sensors; it also presents very interesting and intriguing questions about the underlying physics.

As an explanation for the observed effect, Ghosh et al. ${ }^{1}$ suggested that the dominant mechanism responsible for this highly nonlinear response to the flow could involve a direct forcing of the free electrons in the nanotubes by the fluctuating Coulombic field of the liquid flowing past the nanotubes, through pulsating asymmetric ratchets. The model assumes adsorbed ions which nonetheless do not undergo any average physical drift and needs to invoke ratchets for which there seems to be no independent evidence. Earlier work ${ }^{2}$ had suggested instead that molecular layers of liquid coating the nanotube physically slip along its surface and excite a phonon wind which drags the free carriers in the tube. However, this model would yield an induced voltage linear in the fluid flow velocity. Back in the 19th century, Helmholtz ${ }^{3}$ proposed an electrokinetic mechanism where a voltage induced by a liquid flowing past a solid substrate will appear as a streaming potential involving the ions carried by fluid flow in the diffuse (Debye) layer at the interface, while the mobile charge carriers in the substrate play no role. This mechanism too would, for small flow velocities, predict a linear voltage increase with flow velocity. Our purpose here will be to propose an alternative model capable of explaining the observations-and of making predictions that could be checked by further work-based on flow-induced ion drift.

The experiments of Ref. 1 were performed on nanotube bundles. The nanotubes are micrometer sized in length and nanometric in diameter. Figure 1 shows the induced voltage as a function of the logarithm of the water (average) flow velocity $v_{0}$. The solid line is a fit to the experimental data given by

$$
U=U_{c} \ln \left(\frac{v_{0}}{v_{c}}\right),
$$

where $U_{c}=0.26 \mathrm{mV}$ and $v_{c}=1.54 \times 10^{-7} \mathrm{~m} / \mathrm{s}$.

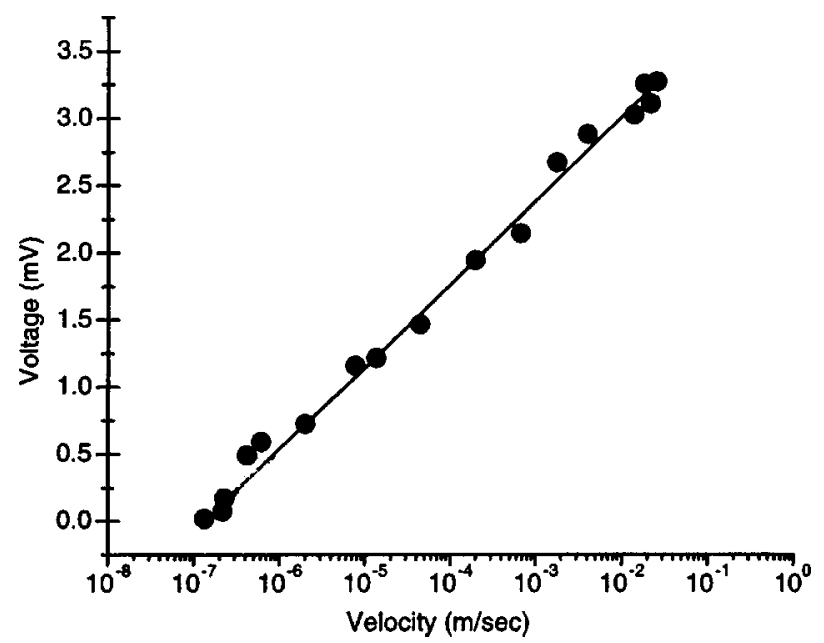

FIG. 1. The voltage $U$ as a function of the logarithm of the (average) fluid velocity $v_{0}$. The solid line is a fit of the form $U$ $=U_{c} \ln \left(v_{0} / v_{c}\right)$. Adapted from Ref. 1 . 


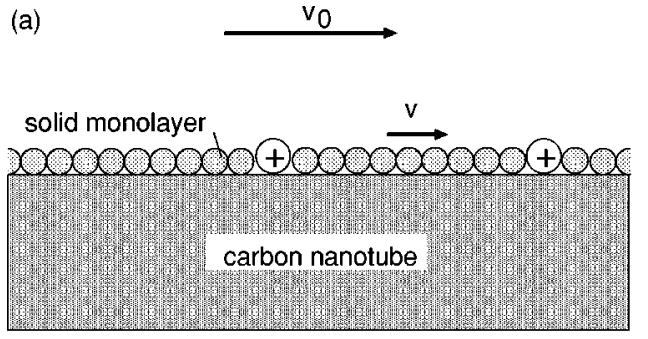

(b)

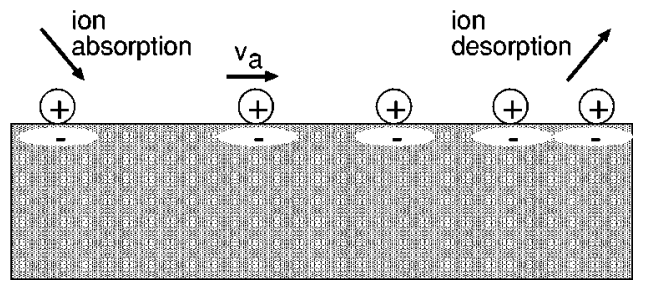

FIG. 2. Carbon nanotube immersed in a flowing liquid. We assume that the liquid molecules nearest the nanotube form a twodimensional (2D) solidlike monolayer, pinned to the nanotube by the adsorbed ions. As the liquid flows, the solid sliding motion along the nanotube is of a stick-slip nature: time intervals of elastic deformation (loading phase) are followed by rapid local yield events. This will result in a force on the ions which increases logarithmically with the fluid flow velocity. The drifting adsorbed ions will produce a voltage in the nanotube through electronic friction against free electrons inside the nanotube. In the stationary state there is a net flow of ions adsorbing on the nanotube upstream and a net flow of ion desorption downstream.

\section{ION STICK-SLIP MODEL}

Although the reasoning is general, let us focus here on water as the fluid and start by assuming that ions dissolved in it become adsorbed on the carbon nanotube surfaces; see Fig. 2. We also postulate that the water molecules in the first layer around a nanotube form an (incommensurate) solidlike monolayer. Recent simulations provided evidence for instances where that does occur ${ }^{4}$. This $2 \mathrm{D}$ solidlike water layer will experience a pinning potential on the nanotube, which is mediated through the adsorbed ions and is in turn dragged or pulled forward by the external liquid water flow. The solid sliding motion along the nanotube occurs by stick-slip motion. Time intervals of elastic deformation (loading phase) are followed by rapid local yield events. During the loading phase the local stress in the 2D solid near an ion will increase linearly with time, until a critical stress $\sigma_{\mathrm{c}}$ is reached, when a local yield (rapid rearrangement process) takes place. We denote the elementary solid element where most of the rearrangement occurs as the "stress block."

A linear (or nearly linear) relationship between driving force and the logarithm of the induced drift velocity has been observed for many systems exhibiting local stick-slip motion-e.g., charge density wave and flux line systems ${ }^{5}$ - as well as for atomic force microscope tips moving in a substrate pinning potential. ${ }^{6}$ These results are collectively understood through a standard picture involving thermally excited jumps over force-biased barriers. ${ }^{7}$ This picture

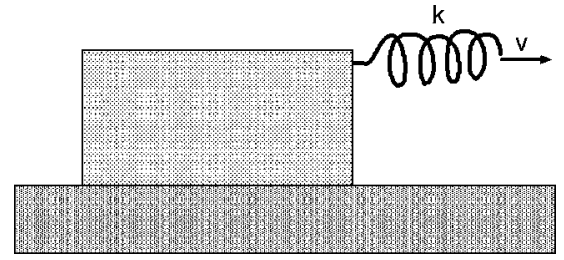

FIG. 3. Mechanical model representing the stick-slip process (loading by elastic deformation followed by abrupt yield) of the 2D solid in the vicinity of an ion [see Fig. 2(a)]. The spring represents the elastic deformation of the 2D solid. When the spring force reaches a critical value (the static friction force) the block starts to slip, reverting back to the pinned state when the spring force approximately vanishes.

will apply for all systems which can be described as effective elastic solids pinned by defects and driven by an external force. The basic picture consists of elastic loading, followed by rapid, local jumps over pinning barriers; in these jumps local regions (stress domains) of the elastic solid move forward. When thermal activation of such a stick-slip process is taken into account, the average stress at yield increases logarithmically with the velocity of the dragging fluid.

Assume that the (average) velocity $v$ of the adsorbed 2D solidlike water monolayer depends linearly on the (average) fluid flow velocity $v_{0}, v=\alpha v_{0}$, as expected for a Newtonian liquid. During the loading phase the strain in a stress block will be of order $u / L$, where $L$ is the linear size of a stress block and $u$ the displacement $u=v t$. The local shear stress in the stress block will be of order $\sigma \approx E u / L$, where $E$ is the elastic modulus of the 2D solid. We define the loading force $F=L a \sigma=E a u=k u$, where $a$ is the thickness of the 2D solid monolayer film and the effective spring constant is $k=E a$. We could expect $E$ to be similar to the elastic modulus of ice-i.e., of order $5 \times 10^{9} \mathrm{~Pa}$-and with $a \approx 0.3 \mathrm{~nm}$ we get $k \approx 1.5 \mathrm{~N} / \mathrm{m}$. Assuming the critical strain at yield to be of order 0.1 we get the critical displacement $u_{\mathrm{c}} \approx 1 \AA$ and the elastic barrier $\epsilon=k u_{\mathrm{c}}^{2} / 2 \approx 0.05 \mathrm{eV}$-i.e., in the same range of energy as a hydrogen bond.

The picture presented above is represented in Fig. 3, where the spring represents the elastic deformation of the $2 \mathrm{D}$ solid. When the spring force reaches a critical value (the static friction force) the block starts to slip and will revert back to the pinned state when the spring force approximately vanishes. This fast slip event corresponds to a yield or rearrangement process of the $2 \mathrm{D}$ solid in the vicinity of the ion. In Refs. 8 and 9 we studied the relationship between the drive velocity $v$ and the (average) spring force $F$ for the model in Fig. 3 including temperature effects. If $F_{\mathrm{c}}$ denotes the "static" friction force (the spring force necessary in order to start the slip of the block), at temperatures $T>0 \mathrm{~K}$ thermal excitation will allow the block to depin before the spring force has reached the critical value $F_{\mathrm{c}}$. The rate of thermal excitation over a barrier $\epsilon$ can be assumed to be of the standard form

$$
w=\nu e^{-\beta \epsilon^{\prime}},
$$

where the prefactor $\nu \approx 10^{10} \mathrm{~s}^{-1}$ and the effective depinning barrier $\epsilon^{\prime}\left(0<\epsilon^{\prime}<\epsilon\right)$ depends on the local shear stress in the 
contact area. By considering the statistical distribution of the microscopic shear stress, the friction versus speed can be calculated. ${ }^{9}$ Three regimes are found, depending on the magnitude of the speed. We define the parameter $v^{*}$ :

$$
v^{*}=\frac{F_{c} \nu}{2 k \beta \epsilon} e^{-2 \beta \epsilon} .
$$

In a wide intermediate range of speeds, where $1 \ll v / v^{*}$ $\ll e^{2 \beta \epsilon}$, we get

$$
F=\frac{F_{c}}{4 \beta \epsilon} \ln \left(\frac{v}{v^{*}}\right) .
$$

In the extremly low velocity limit, where $v / v^{*} \ll 1$, we have

$$
F=\frac{k v}{\nu} e^{\beta \epsilon} .
$$

For very high velocity instead, $v / v^{*} \gg \exp (2 \beta \epsilon)$, we have, on the other hand,

$$
F=\frac{F_{\mathrm{c}}}{2}\left(1-\frac{v^{*}}{2 \beta \epsilon v} e^{2 \beta \epsilon}\right) .
$$

We now assume that the force $F$ will also act on the ion which is at the same time embedded in the $2 \mathrm{D}$ solid monolayer and adsorbed on the nanotube. This force will result in a slow average drift motion of the ion along the water flow direction. Since $F$ is very small, linear response should apply. The adsorbed ion drift velocity is thus

$$
v_{a}=\mu F,
$$

where the mobility $\mu$ depends on the lateral ion diffusion barriers. Combining Eqs. (2) and (3) gives

$$
v_{a}=v_{a}^{c} \ln \left(\frac{v_{0}}{v_{c}}\right) \text {, }
$$

where $v_{c}=v^{*} / \alpha$ and

$$
v_{a}^{c}=\frac{\mu F_{\mathrm{c}}}{4 \beta \epsilon} .
$$

If we write $F_{\mathrm{c}}=k u_{\mathrm{c}}$, where $u_{\mathrm{c}}$ is the displacement necessary in order to induce the yield, we get from Eq. (1)

$$
v_{c}=v^{*} / \alpha=\frac{u_{\mathrm{c}} \nu}{2 \alpha \beta \epsilon} e^{-2 \beta \epsilon} .
$$

Note that Eq. (5) can be written as

$$
2 \beta \epsilon=\ln \left(\frac{u_{c} \nu}{2 \alpha \beta \epsilon v_{c}}\right),
$$

which shows that $\epsilon$ is very insensitive to the exact value of $\alpha, u_{c}$, and $\nu$. If we assume $\alpha=0.1, u_{c} \approx 1 \AA$, and $\nu$ $\approx 10^{10} \mathrm{~s}^{-1}$, we get with (from Fig. 1) $v_{\mathrm{c}}=1.54 \times 10^{-7} \mathrm{~m} / \mathrm{s}$, $\epsilon \approx 0.2 \mathrm{eV}$, which is very plausible.

\section{FLOW-INDUCED CURRENT AND VOLTAGE: ELECTRICAL PROPERTIES}

Assume that there is a $2 \mathrm{D}$ concentration $n_{e}$ of conduction electrons in the carbon nanotube. We shall use here a semi- classical picture for the motion of the electrons. Under the ion-originated external forces the conduction electrons drift with the velocity $v_{e}$, so that the electric current in a nanotube will be $I=2 \pi r n_{e} e v_{e}$, where $r$ is the radius of the nanotube. The equation of motion for the drift velocity is given by

$$
m_{e} \dot{v}_{e}=-m_{e} \frac{1}{\tau} v_{e}-m_{a} \eta\left(v_{e}-v_{a}\right) \frac{n_{a}}{n_{e}}+e E,
$$

where $\tau$ is a Drude relaxation time, $\eta$ an electronic friction coefficient, $n_{a}$ the concentration of adsorbed ions, $E$ the electric field in the 2D nanotube, and $v_{a}$ the drift velocity of the adsorbed ions. In deriving Eq. (7) we assumed that the frictional drag from the moving adsorbates to be distributed uniformly over all the conduction electrons in the nanotube. The electronic friction coefficient $\eta$ can be related to the adsorbate-induced increase in the nanotube resistivity. ${ }^{9}$ Thus, when $v_{a}=0$ (no fluid flow) we get in steady state

$$
v_{e}=\frac{e \tau E / m_{e}}{1+\eta \tau\left(m_{a} / m_{e}\right)\left(n_{a} / n_{e}\right)},
$$

so that the current

$$
I=\frac{2 \pi r n_{e} e^{2} \tau E / m_{e}}{1+\eta \tau\left(m_{a} / m_{e}\right)\left(n_{a} / n_{e}\right)} .
$$

Thus, $U=R I$, where the resistivity

$$
R=R_{0}\left(1+\eta \tau \frac{m_{a}}{m_{e}} \frac{n_{a}}{n_{e}}\right)=R_{0}(1+\kappa),
$$

where $R_{0}$ is the nanotube resistivity in the absence of adsorbates:

$$
R_{0}=L m_{e} /\left(2 \pi r n_{e} e^{2} \tau\right) .
$$

We consider now the full equation (7) in two different limiting cases: namely, short circuit and open circuit. In the first case $E=0$ so that in the stationary case Eq. (7) takes the form

$$
0=-m_{e} \frac{1}{\tau} v_{e}-m_{a} \eta\left(v_{e}-v_{a}\right) \frac{n_{a}}{n_{e}},
$$

so that

$$
v_{e}=\frac{\left(m_{a} / m_{e}\right)\left(n_{a} / n_{e}\right) \eta \pi v_{a}}{1+\left(n_{a} / n_{e}\right)\left(m_{a} / m_{e}\right) \eta \tau}
$$

and thus

$$
I=\frac{2 \pi r n_{a}\left(m_{a} / m_{e}\right) \eta \tau e v_{a}}{1+\kappa}=\frac{\kappa}{1+\kappa} 2 \pi r n_{e} e v_{a} .
$$

The second limiting case (open circuit) corresponds to $v_{e}$ $=0$ so that, from Eq. (7), $U=L E$ is given by

$$
U=\frac{L m_{a} \eta}{e} \frac{n_{a}}{n_{e}} v_{a}=\kappa R_{0} 2 \pi r n_{e} e v_{a} .
$$

We expect $\kappa>1$ so that for the short-circuit case the current $I \approx 2 \pi r n_{e} e v_{a}$ is nearly equal to the current expected if the nanotube conduction electrons drift with the same velocity as the adsorbed ions.

We note that $R$ given above represents the internal resis- 
tance of the nanotube bundle as an electromagnetic force (EMF) generator. The measured resistivity $\rho_{0}$ of the nanotube bundles in the normal atmosphere is $\rho_{0}=0.02 \Omega \mathrm{m}$ so that the resistance $R_{0}=\rho_{0} L / A$ where the length $L=1 \mathrm{~mm}$ and the cross section area $A=0.4 \mathrm{~mm}^{2}$. Thus $R_{0}=50 \Omega$. If the electron flow velocity $v_{e}$ is measured in $\mathrm{m} / \mathrm{s}$ and if the electron concentration $n_{e}=2 \times 10^{27} \mathrm{~m}^{-3}$, we get the electron current in amperes,

$$
I=A n_{e} e v_{e} \approx 100 v_{e}
$$

and the induced voltage in $\mathrm{mV}$,

$$
U=R_{0} I=5 \times 10^{6} v_{e} .
$$

The lowest flow velocity for which a flow-induced voltage was measured was $2 \times 10^{-7} \mathrm{~m} / \mathrm{s}$ and in that case the induced voltage was about $0.2 \mathrm{mV}$. Now, this is basically the value one would obtain if the drift velocity of the electrons in the nanotubes equaled the (average) liquid flow velocity and if the resistivity $R$ for the liquid-immersed nanotube bundles were the same as that of the bundles in the atmosphere. Thus, with $v_{e}=2 \times 10^{-7} \mathrm{~m} / \mathrm{s}$, Eq. (10) gives

$$
U=1 \mathrm{mV} .
$$

This is only 5 times higher than the measured voltage, indicating (if as we expect $R$ is similar to $R_{0}$ ) that the electron drift velocity in the nanotubes, in the limiting case of the lowest possible fluid flow velocity where the induced voltage can be observed, is of order the average fluid flow velocity. At higher fluid flow velocity drag is less perfect, and this is no longer the case, since the voltage increases logarithmically with the fluid flow velocity. At the highest studied fluid flow velocity $v_{0} \approx 10^{-2} \mathrm{~m} / \mathrm{s}$ the electron drift velocity would be roughly a factor of $10^{5}$ lower than the average fluid flow velocity.

\section{CONSEQUENCES OF THE MODEL}

Because in our stick-slip model the logarithmic voltage increase with flow velocity is due to the thermally activated jump of the ions, biased by the force arising from the drift motion of the solid monolayer, we can anticipate first of all a strong temperature dependence. This is apparent in Eq. (4a) and (4b) above, where the strongest temperature dependence is derived from the thermal Boltzmann factor which occurs in the microscopic expression for the mobility $\mu$, which should thus increase exponentially with temperature. In addition there is also a linear temperature prefactor $1 / \beta \sim T$, but this is in most cases negligible compared to the Boltzmann factor. There may also be a temperature dependence arising from a temperature dependence of the concentration of adsorbed ions. In any case, a strong temperature dependence of the induced voltage is predicted by our model and should provide a first possibility of experimental test.

A second aspect, which strongly distinguishes our model from fluctuating ratchets, is the flow-induced concentration of ions from upstream to downstream. In the free fluid, every ion is neutralized by some counter-ion of opposite charge. When an ion-say, positive-adheres upstream to the nano- tube surface, it will get to a large extent neutralized by the image electron charge in the nanotube. The corresponding negative counter-ion in the fluid is no longer neutralized. Being made redundant by the positive-ion adsorption, it must go somewhere else. When, on the other hand, after having drifted along a nanotube the positive ion leaves the nanotube surface some distance downstream, it will lose its neutralizing image electron, and must at that point again recuperate a counter-ion. This suggests that the flow-induced drag of positive adsorbed ions will be accompanied by the simultaneous flow of image electrons inside the nanotube and by a drift of negative counter-ions inside the fluid, which will migrate from upstream, where they are redundant, to downstream, where they are needed. In that case we have two negative currents, one electronic inside the nanotube and one ionic, due to the negative counter-ions, against only one ionic positive current, that of the adsorbed ions. The end result is a net negative current, as observed.

This outcome differs from that of models such as that by Ghosh et al., ${ }^{1}$ where no physical drift of ions is involved. If, as we suggest, ionic transport is involved, then there will not generally be a spatially uniform steady state. To be sure, there could be a nonuniform steady state where the ion concentration (of both signs) is slightly higher downstream than upstream. Depending on geometrical conditions, flow velocity, etc., the ions might or might not have time to diffuse backwards upstream, against the flow, so as to establish such a steady-state equilibrium. That suggests the possibility to look in principle at that ion concentration gradient as the possible signature of an ion drift mechanism, although it is at the moment unclear whether such a gradient could in fact be measured.

A third consequence of our model is the presence of characteristic stick-slip noise, which should be readily observable by frequency analysis of the induced voltage. Straightforward as this seems, it might constitute the most direct and simplest test of the model proposed.

\section{SUMMARY AND CONCLUSIONS}

We propose that the logarithmic voltage observed in nanotubes bundles upon immersion in a flowing liquid with ions dissolved could have the same thermal barrier jump origin as that observed for frictional stick-slip processes between solids. Once ions adsorb on the nanotubes, they should be dragged not just by flowing individual liquid molecules, but by some kind of solid layer of molecules which surrounds the nanotube and which is rigid enough to support the stick-slip motion. Each drifting adsorbed ion can in turn drag along electrons inside the nanotube, through the very same electronic frictional force which enables a fixed adsorbed ion to cause electrical resistance inside the nanotube.

By assuming this mechanism to be at work we obtained a very simple formula for the nanotube generator electrical properties. Expressions for the internal resistance, shortcircuit current, and open-circuit voltage obtained in this manner seem entirely reasonable. Moreover, the latter quantities increase logarithmically with the flow velocity, as is seen experimentally. 
Various tests can be considered for our mechanism. First, the logarithmic voltage increase should be strongly temperature dependent. Second, the physical dragging of adsorbed ions should cause the ion concentrations in the fluid to become nonuniform and, in particular, to become higher downstream. Third, a characteristic stick-slip noise spectrum should arise on top of the dc-flow-induced voltage. It is hoped that these preliminary simple predictions will stimulate further experimental work on this intriguing effect.

\section{ACKNOWLEDGMENTS}

We are much indebted to Professor N. Kumar and Professor A.K. Sood for introducing us to their new effect, for making unpublished information available to us, and for illuminating discussions. Work at SISSA was sponsored through INFM PRA NANORUB, through MIUR COFIN 2003, as well as FIRB RBAU01LX5H and FIRB RBAU017S8R, and by Regione Friuli Venezia Giulia. One of us (B.N.J.P.) is grateful to the International Centre for Theoretical Physics, where this work was initiated, for hospitality. B.N.J.P. also thanks the EC for a "Smart Quasicrystals" grant under the EC Program "Promoting Competitive and Sustainable Growth." This research was supported by the National Science Foundation under Grant No. PHY9907949 .
${ }^{1}$ S. Ghosh, A. K. Sood, and N. Kumar, Science 299, 1042 (2003).

${ }^{2}$ P. Kral and M. Shapiro, Phys. Rev. Lett. 86, 131 (2001).

${ }^{3}$ H. L. F. van Helmholtz, Ann. Phys. (Leipzig) 7, 337 (1879).

${ }^{4}$ M. Odelius, M. Bernasconi, and M. Parrinello, Phys. Rev. Lett. 78, 2855 (1997); R. Zangi and A. E. Mark, ibid. 91, 025502 (2003).

${ }^{5}$ See, e.g., P. W. Anderson, Phys. Rev. Lett. 9, 309 (1962); G. Mihaly and L. Mihaly, ibid. 52, 149 (1984).

${ }^{6}$ E. Gnecco, R. Bennewitz, T. Gyalog, Ch. Loppacher, M. Bammerlin, E. Meyer, and H.-J. Günterodt, Phys. Rev. Lett. 84,
1172 (2000); E. Riedo et al., ibid. 91, 084502 (2003).

${ }^{7}$ B. N. J. Persson and A. I. Volokitin, Surf. Sci. 310, 314 (1994); B. N. J. Persson, J. Chem. Phys. 98, 1659 (1993); Sliding Friction: Physical Principles and Applications (Springer, Berlin, 2000).

${ }^{8}$ B. N. J. Persson, O. Albohr, F. Mancosu, V. Peveri, V. N. Samoilov, and I. M. Sievebaek, Wear 254, 835 (2003).

9 B. N. J. Persson, Phys. Rev. B 51, 13568 (1995); 61, 5949 (2000). 